\title{
The Construction and Collapse of Stardoms Characteristics from the Perspective of Network Communication
}

\author{
Yening You*
}

\author{
JiShou University, Xiangxi, 416000, China \\ *Corresponding author. Email: jzhouyou@163.com
}

\begin{abstract}
With the development of the times and the progress of new media technology, the dissemination of all kinds of information breaks through the limits of time and space, the distance between stars and fans is getting closer and closer. In order to obtain more audiences' attention and the economic value brought by audiences' attention, stars chose to build their characters. Stars have a certain influence on society, especially teenagers. If stars' characteristics are properly constructed and disseminated, it will bring positive impact to society, once characteristics built by stars begin collapsing, it will interfere with the normal social order, disrupt the correct public value judgment. This article starts from the perspective of network communication, discusses the causes, methods and influence of the destruction of human beings.
\end{abstract}

Keywords: Stars' characteristics, Network communication, Fans economy.

\section{INTRODUCTION}

'characteristics' means the setting of the character image, which is a professional vocabulary used in comics, cartoons and films, including the personality characteristics, appearance characteristics, character modeling and so on [1]. 'the collapse of characteristics' is originated from 'characteristics'. It was first derived from the 387th episode of the Japanese animation NARUTO. The sudden change in the painting style of comic characters makes the audience cry 'characteristics collapse'. Through then, 'the collapse of characteristics' was wieldy used to describe the image changes of virtual characters, including appearance and character. [2] With the increasing people to use this term, the term is used wider and wider, especially in the entertainment world. 'stars' characteristics' usually refers to the images that are intentionally magnified. 'The collapse of the stars ' characteristics' means that the performance of the stars is not consistent with the image created, and the image is subverted. It often refers to the fact that the public image of the celebrity set by the agent is not in place. On the other hand, it means that someone's reputation is destroyed by something, destroying the public's healthy and positive impression of him.
The network dissemination actually refers to through the computer network human information (including news, knowledge and so on information) dissemination activity [3]. In the network communication environment, the information received by the audience is more diversified and the manifestation is more three-dimensional. There are more stages for stars to show their characteristics, and the distance between fans is closer. In this social environment, the construction and collapse of stardoms' characteristics becomes easier and faster.

\section{REASONS OF CHARACTERISTICS CONSTRUCTION}

The basic purpose for stars to build their characteristics is to enhance their commercial value. Richard Dyer refers that stars are 'constructed individual' that is constructed by media texts such as films and mass media in STARS [4]. In this regard, stars are a commodity, and the audience is consumers. Jean Baudrillard points that in consumer society, people consume more at the symbolic level than at the material level. Before consumption, commodities are simply in symbolic state, and the value of goods is determined by 
the symbol [5]. So, characteristics make stars difference to attract audience to increase their commercial value.

\subsection{Differentiation Positioning}

At first, stars' characteristics are only a single personality label. Like, 'square-toes', 'flat attributable', 'Four Thousand Years Beauty' and so on. However, with more and more stars began using this strategy, a large number of similar and single characteristics lead to aesthetic fatigue. Therefore, they cannot be distinguished from different stars to improve their identification. As a result, stars' characteristics are going to become diversification and multi-polarization. Some characteristics like 'lucky dog', 'surfing internet in 16g' which are originated from internet buzzword are showing in the stage. At the same time, image settings that are contrary to personal traits also appeared. Female celebrities in the entertainment industry sometimes establish the image of a "foodie". The female celebrities who need to strictly control their diet to maintain their figure like to eat and drink milk tea, in order to establish emotional connections with the audience. DILIREBA who is a actress with cool and powerful temperament acts in a simple and ignorant role in a TV series, to shape the "cute" character on the screen. This contrast gives the audience a sense of intimacy, also let this characteristic no longer model, but only belong to DILIREBA, distinguished from other stars.

\subsection{Attract Brand Owner}

Branders usually choose stars similar to their own brand tonality to represent. Li Sun created 'hot mother' and 'good at nourishing of life' those two characteristics through her TV series hot mothers as well as the dynamic shaping of hot mothers and life-loving images on Weibo, and received represent such as Doubendou and Hersda Cooks. Lei Huang got Alien Integrated kitchen's represent by setting up 'chef Huang' characteristic in slow variety show The life you aspire to and 'the Pacifier' in dad, where are we going. So we can see stars build their own characteristic can attract brand owner. In the consumer society, the marked characters are more likely to leave a deep impression on the audience. Seeing their endorsement of commercial advertising, the psychological sense of identity is triggered naturally to cause consumer impulse [6].

\section{SOURCES OF STARS' CHARATERISTICS}

\subsection{Company Shaping}

Star agencies usually choose to build stars characteristics intentionally to make distinct to other competitors. When fans buy related products endorsed by a celebrity, it is not for the celebrity or not just to obtain the product. Some fans even buy products that they do not need at all. In fact, fans are paying for the psychological satisfaction created by idols. The agency needs the support of a huge fan market, so it will create stars that satisfy fan fantasy for fans. In the era of social networks, stars' characteristics is a long-term image of the star 's operating team, which conveys to the social media public a kind of labeled thinking concept in line with the public's aesthetics [7]. With the development of network communicational technology, stars have many channels to show their characteristics, they are no longer choose to be mysterious. They usually make direct and bilateral interaction with fans by using social platforms such as Weibo and Tik Tok, which can strengthen their characteristics.

\subsection{ENHANCE AND REPRODCTION BY FANS}

The psychological projection of fans to idols is one of the reasons for the emergence of "characteristics". Through the observation of idol works and the interactive observation of the real world, fans constantly improve the image of idols in their hearts, and unconsciously reflect their attitudes, desires or personality characteristics on their favorite idols, which can be said to be fan's self-imagining projection. Distributed network structure forms a 'decentralized' new media structure, with everyone involved in production and creation [8]. Fans no longer just simply watch the text like songs or episodes created by stars, but also can participate their own contents. Fans have great autonomy, and can re-clip and combine these texts, make the stars form unique characteristic which fans love to see. Personalized services allow the content produced by these fans to be delivered to the target group more accurately and quickly. And these text productivities made by fans has it specialty and professional. American scholar Fresk's research focuses on fans' 'textual productivity ' and thinks it has the same influence as official texts. Fan text productivity is the closest to the artistic productivity confirmed by official culture.

\section{REASONS OF CHARACTERISTICS COLLAPSE}

Canadian sociologist Erving Goffman thinks everyone in daily life is an actor, he split stage for people to perform in 'foreground' and 'background' two regions. 'foreground' is for performers to perform, and 'background' is for performers to do some prepare and rest. The foreground and background can be switched instantaneously [9]. And in stars research, 'foreground' is for stars to show their characteristics for audience, 'background' is the real character the star has. If the 'background' was shown to the audience, audience will be disappointed and the perform will be cut. When 
celebrity idols are exposed to backgrounds that run counter to the ideal personality that has been formed, such as drug abuse, drunk driving and other criminal behaviors, cheating, pornographic photos, lying and other moral issues, it may quickly become a hot topic and have a series of impacts. For example, it is more difficult for the public to accept that celebrities with criminal or moral problems appear in active film and television images, or they have obvious resistance to their participation in commercial activities and other public behaviors.

In theater theory, Goffman divides people's performance areas into "foreground" and "background". The foreground is a place where people perform, and what they show is a socialized self. The background is the place where the front stage performers are prepared, and the audience is not allowed to see it. In the backstage, what people express is the most essential part of themselves. Network communication technology gives stars more chance to show themselves, but also makes the boundary of 'foreground' and 'background' fuzzy, which easily lead to background becomes foreground. As a natural person, celebrities have the right to privacy in their backstage life, but while celebrities benefit from attention resources, it is also difficult to avoid exposure of their private lives brought about by attention effects. The information retained by social media is easy to search. Anyone can dig out a dynamic shared by social media ten years ago with the intention. With the increase in the channels for the media to obtain information, this kind of viral radiation spreads at an alarming speed, and the flow of information is greatly enhanced. If an idol individual makes a behavior that violates his own characteristic and is caught, it will be infinitely amplified and spread. The set is destroyed or even completely destroyed. Sharing daily life in social media like Weibo will expose stars characteristic flaws, giving a live in some media may let stars out of company's control and show their real-me. For example, Tianlin $\mathrm{Qu}$ always been known as "outstanding student", but in a live he asked "what is CNKI?", which made netizens questioned his doctoral degree and searched the Internet to find his academic fraud.

\section{INFLUENCE OF CHARACTERISTICS CONSTRUCTION}

As public figures, stars have high popularity, which will influence ideology of their fans. A positive characteristic will bring positive influence, lead fans to take part in programs for public good and pass on correct values. If a star constructs a positive characteristic, such as "caring for small animals", "caring for children", and "being willing to do charity", then all his actions must be aimed at maintaining these images. His fans will also do some charity together to follow in his footsteps. For fan groups, there are often fan groups doing charitable activities in the name of a star. All these have a positive impact on social development and the growth of young people.

But if a star just relay on his characteristic without any achievement, he will bring unhealth tendences to the entertainment circle and even the whole society. At the moment when the characteristics collapses, fans will feel deceived and feel lost and angry. In their daily lives, they may also have doubts about human nature. If some well-behaved celebrities are unknown, and others with bad reputations are touted because they are good at character construction, it will undoubtedly make some celebrity teams think that as long as they construct a popular characteristic, everything can be forgiven. If a people can win attention with adorable a characteristic, there will be no one want to improve themselves and completely becomes a commodity without self-awareness, which will disturb their judgement about public value.

\section{CONCLUSION}

In the era of Internet communication, the demand side has caused a reversal and drastic change in the power of young audiences in the diachronic dimension. The abundance of selectable resources has increased significantly, and the convenience of media content and channel intervention has been greatly improved in both directions. What is really scarce is the value of stars characteristics resources. From a technical perspective, the capacity of celebrity resources in Internet media is infinitely rich, but not all characteristics can be effectively disseminated and have the possibility of being accepted and consumed by the audience. Whether a star's characteristics can ultimately be accepted by the audience, realize consumption value, economic value, and produce social impact requires reasonable operating rules and value judgments. Under the network communication environment stars' characteristics can be diffused and be disclose easily. As a result, when constructed their characteristics, stars should pay attention that the difference between characteristics and their real-me should not be too big. Don't rely on economic benefit bring by characteristic but ignore their own improvement. Only working hard to tap one's own advantages and talents and create outstanding representative works is the long-term solution to retain fans and the audiences.

\section{REFERENCES}

[1] Xiaoqin Fan, Communication Interpretation of 'characteristics collapse', Today's Massmedia, 2018, 26(03):49-50.

[2] Qiongyao Wang, The construction and collapse of star 'characteristics' under the theory of drama 
simulation, RADIO \& TV JOURNAL, 2019, (08): 107-108.

[3] Wenbo Kuang, Introduction to Network Communication, 3rd ed., Higher Education Press, 2009.

[4] Richard Dyer, Paul Mcdonald, Stars, new ed., British Film Institute, 1998.

[5] Yaxin Zhang, Chen An, Adilai Aymetiniyazi, Journal of News Research, 2020, 11(02):10-13.

[6] Xiaoqin Fan, Communication Interpretation of 'characteristics collapse', Today's Massmedia,
2018, 26(03):49-50.

[7] Xin Zhang, Yanning Wei, Character and Marketing Analysis of Stars in the Era of Social Network, Home Drama, 2020(02): 76+78.

[8] Lan Peng, Introduction to network communication, 3rd ed., China Renmin University Press, 2012

[9] Erving Goffman, The Presentation of Self in Everyday Life, 1st ed., Anchor, 1959 